\title{
Solar-vortex power plants: Principles of effective work and technical requirements on the preparation of initial data for design
}

\author{
Alexander Solovyev ${ }^{1, *}$, Dmitry Solovyev ${ }^{2,3}$, and Liubov Shilova ${ }^{4}$ \\ ${ }^{1}$ MV Lomonosov Moscow State University, Faculty of Geography, 19991, GSP-1, Moscow, Lenin \\ Hills, d. 1, Bldg. 19 \\ ${ }^{2}$ Shirshov Institute of Oceanology, Russian Academy of Sciences, 117997, Nahimovskiy prospect 36, \\ Moscow, Russia \\ ${ }^{3}$ Joint Institute for High Temperatures, Russian Academy of Sciences, 125412, Izhorskaya st. 13 \\ bd.2, Moscow, Russia \\ ${ }^{4}$ Moscow State University of Civil Engineering (National Research University) (MGSU), 129337 \\ Yaroslavskoye Shosse 26, Moscow, Russia
}

\begin{abstract}
This article discusses the conceptual problems of the energy conversion process from radiant radiation into the energy of flows and electric energy in solar convective-vortex power plants. Moreover, this research includes the analysis of the factors that can contribute to the achievement of the optimal conditions for the control of the thermal energy concentration processes of solar radiation into the energy of tornado-like flows. Also, this article will discuss the results of the development of appropriate technical requirements for the design and construction of the building foundations and structures of solar-vortex power systems.
\end{abstract}

\section{Introduction}

The development of basic renewable energy concepts that are based on the needs of environmental safety of energy technologies, real prospects for the depletion of fossil resources and the diversification of renewable sources, is attaining great importance in modern environmental science. Among the variety of the renewable energy systems, that lack the definition of conceptual provisions, it is crucial to highlight a relatively nonadvanced technology for converting the infrared component of solar radiation into the flow and electric energy.

In the technology described in this article, conditions for the localization of heating in the surface layer are obtained by utiliztation of unidirectional permeability through the collector surface of the short-wave component of solar radiation. If there is a vertical temperature gradient in the exhaust pipe located in the center of the solar collector, then the gravitational instability of the air masses is formed; thus leading to an upward convective

\footnotetext{
* Corresponding author: a.soloviev@geogr.msu.ru
} 
flow. The energy of this stream is converted into electricity by means of a wind wheel connected to the electric generator shaft.

The efficiency parameter of thermal energy conversion into the energy of air masses motion is determined by the Carnot principle. In accordance with this principle the power of the generated flows is directly proportional to the size of the collector, the height of the pipe and the vertical temperature difference therein. The thermodynamic limit of the solar energy conversion into energy of flows, is determined by the second law of thermodynamics for closed equilibrium systems, acts as an obstacle to the practical implementation of this technology. (significantly limits the practical feasibility of technology). A possible solution to the problem of increasing the energy output of thermal energy towards flows can be achieved by creating conditions under which the energy conversion in the "heat energy flows-flow" system is carried out with nonequilibrium, irreversibile and open transfer processes. This makes it important to consider the basic concepts of the flow thermal energy conversion initiated by solar radiation, which must be based on the principles of nonequilibrium thermodynamics. In this paper we discuss the extent to which taking into account the nonequilibrium and irreversibility of energy exchange processes can lead to the possibility of creating effective methods for overcoming the limiting values of mechanical work in the aerodynamic technology of solar energy conversion. In connection with the urgency of the practical implementation of the proposed concept for the creation of solar-vortex power plants, the results of the development of the corresponding technical requirements that are necessary for the design and construction of the building foundations and structures of solar-vortex power systems are also presented.

\section{Efficiency of solar-wind power plants}

To estimate the possibility of the flow intensification in a solar-greenhouse power plant with interconnected vertical and horizontal convectionally swirling flows, the following model for converting radiant-heating energy into the kinetic energy of air currents is considered (Figure 1).

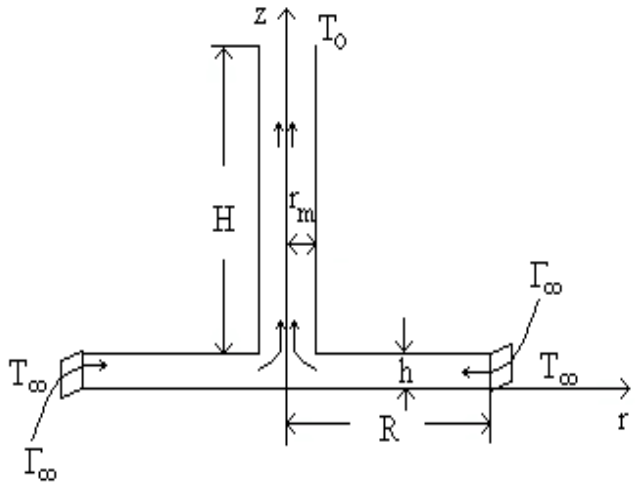

Fig. 1. The scheme for converting the energy of radiant heating of the surface layer into the energy of a convective swirling flow. Ambient temperature $-\mathrm{T}_{\infty}$; temperature of the underlying surface of the collector $-\mathrm{T}_{0}$; peripheral circulation of speed $-\Gamma_{\infty}$; radius of the heated surface layer $-\mathrm{R}$; radius of a convective vortex $-r_{m}$; height of surface heated layer $-h$.

Surface collector creates conditions for the concentration of the infrared component of electromagnetic radiation and air heating inside. Vertical pressure drop in the cylindrical pipe on the axis of the cylindrical collector forms axially symmetric flows converging 
towards the center from the ascending convective flows. An angular momentum is applied to the outside air entering the collector at the lateral boundary. A non-uniform horizontal temperature drop in the reservoir initiates hydrodynamic perturbations leading to centrifugal convection with a nonzero speed rotor, due to which the heated air moving from the reservoir to the pipe is replaced. The rotating surface flow in the tube is concentrated in the mechanical energy of the air convection flow [1], the formation mechanism of which can be compared with the phenomenon of "tornado".

One of the crucial issues that are encountered in the development of power plants using renewable energy sources is a relatively low specific power per unit of occupied space [2]. Such stations can be considered effective only if the energy generation density is not less than $50 \mathrm{~W} / \mathrm{m}^{2}$. This condition is met only by sources of atomic energy with a density of $150 \mathrm{~W} / \mathrm{m}^{2}$ [3]. Compared to the solar radiation density of $1000 \mathrm{~W} / \mathrm{m}^{2}$, the "Solar Chimney" type of power plants are characterized by relatively low values of the specific power of the solar energy transformer of the order of $10 \mathrm{~W} / \mathrm{m}^{2}[2,4]$.

This happens due to the fact that the process of wind transformation of the radiant heating energy of the medium into the energy of flows is performed in constructions that create the conditions of equilibrium thermodynamics and linear hydrodynamics. This follows from the consideration of the process of converting solar energy into mechanical energy of air convective flows [1]. Under equilibrium conditions of the medium, the amount of heat is expended on heating a unit $\tau_{1}$ of the air volume density $\rho_{1}$ from temperature $T_{\infty}$ to temperature $T_{0}$ in the surface layer:

$$
\mathrm{Q}=\mathrm{C}_{\mathrm{p}} \rho_{2} \tau_{1}\left(\mathrm{~T}_{0}-\mathrm{T}_{\infty}\right)
$$

where $\rho$ is the density of air after heating in the isobaric process, $C_{p}$ is the heat capacity. The lifting force, heated and expanded to the volume of $\tau_{2}=\tau_{1} T_{0} / T_{\infty}$ air mass, is:

$$
F=\left(\rho_{2}-\rho_{1}\right) g=\rho_{1} g \tau_{1} \frac{T_{0}-T_{\infty}}{T_{\infty}} .
$$

The work of this force when moving the air to a height $\mathrm{H}$ :

$$
\mathrm{A}=\rho_{1} \mathrm{~g} \tau_{1} \frac{\mathrm{T}_{0}-\mathrm{T}_{\infty}}{\mathrm{T}_{\infty}} \mathrm{H}
$$

The coefficient of conversion $\eta$ that brought to the flow of thermal energy in the work on the movement of air particles is represented by the ratio:

$$
\eta=\frac{\mathrm{A}}{\mathrm{Q}}=\frac{\mathrm{gH}}{\mathrm{C}_{\mathrm{p}} \mathrm{T}_{\infty}} .
$$

Taking into account $\mathrm{C}_{\mathrm{p}}=1000 \mathrm{~J} / \mathrm{kg}^{2} ; \mathrm{g}=9.8 \mathrm{~m} / \mathrm{s} 2 ; \mathrm{T}_{\infty}=300 \mathrm{~K}$; height $\mathrm{H}=200 \mathrm{~m}$, we obtain value for the coefficient of thermal energy conversion into convective airflow energy $\eta=0,65 \%$. On the other hand, taking into account the energy dissipation of convective ascending flows into heat by viscous and turbulent friction will lead to a decrease in the energy conversion coefficient.

The value of the solar energy conversion coefficient (1) is obtained under the condition that forces the flow to be formed only by vertical convection. Therefore, the achievement of economically feasible output capacity can be associated with the use of a relatively high pipe. That is why a $1000 \mathrm{~m}$ pipe is developed in the project of the Australian power plant [5]. Meanwhile, such a solution to the problem of intensification of thermally rising flows initiated by IR heating of the surface layer on the solar collector is not univocal. Since it 
initially ignores turbulent horizontal convective flows caused by temperature differences inside and outside the reservoir, as well as energy transfer by vorticity of the flow entering the collector. Essentially speaking, both the Spanish and Australian projects of the solargreenhouse power plant connect the generation of artificial wind energy with the linear upward flow model inherent in natural thermal. The flows forming in the power plant should rather be considered as a prototype of a tornado. Energy of this tornado is determined not so much by the vertical stream, as by the vorticity of the horizontal air-mass spirally converging toward the center, which are created on nonlinear instabilities.

The magnitude of the coefficient of hydrodynamic transformation of solar energy initiated by the greenhouse effect can be controlled by selecting the optimum values for the swirling angle of the flow, the height of the collector, its radius, and the height and radius of the exhaust pipe [6..8]. It is important that, from the point of energy transfer view, the vortex flow is of an order of magnitude more efficient than a convectional flow [9]. In the solar-vortex power plant with the characteristics of the Spanish station, the coefficient of efficiency calculated according to the formula (4) is $6.5 \%$. The optimal conditions for the operation of greenhouse solar energy converters should first of all be determined from the maximum realized height of the collector, ensuring high air temperature, [10..11]. In addition to using the external curvature of the flow entering the collector for this purpose, other ways of extending the path of the air mass can not be excluded from consideration. Including, the passage of air through the system of circulation cells, organized inside the reservoir. The creation of a cellular structure of the flow can be achieved with the help of inhomogeneous spatial localization of concentrated solar radiation penetrating into the collector.

\section{About the technical requirements for the preparation of initial data for the design of the building foundation and structures of solar-vortex power systems}

The results of engineering-geological and hydro-geological studies, stated in the survey report, should contain information on the location of the proposed construction site, its climatic and seismic conditions, and the results of studies of soils and groundwater.

We need information about the engineering-geological structure and lithologic composition of the soil thickness and on the observed unfavorable physico-geological and other phenomena (karst, landslides, subsidence and swelling of soils, mine workings, etc.).

For the preparation of design documentation for the construction of the station, data about hydrogeological conditions is required, indicating the altitude marks of the emerged and established levels of groundwater, the amplitude of their fluctuations and the water discharge; about the presence of hydraulic connections of the water horizons with each other and the nearest open water bodies, as well as information about the water aggressiveness in relation to the materials of foundation constructions .;

In the data necessary for design, information should be provided about the soils of the construction site, including a description in the stratigraphic sequence of the basement bedding, the form of the occurrence of subterranean formations, their dimensions in terms of depth, age, origin and classification names, composition and condition of soils.

For the selected layers of soil, physical and mechanical characteristics should be given, including: soil density and humidity; coefficient of soils' porosity; granulometric composition for coarse clastic and sandy soils; - number of ductility and yield index; angle of internal friction, specific cohesion and modulus of deformation of soils; coefficient of filtration; coefficient of consolidation for water-saturated silty-clay soils with a yield index exceeding 0.5 ; time resistance to uniaxial compression, coefficient of softening; the degree 
of salinity and solubility for rocky soils; relative subsidence, as well as the initial pressure and initial critical humidity for subsidence soils; relative swelling, swelling pressure, and linear shrinkage for swelling soils; coefficient of weathering for eluvial soils; quantitative and qualitative composition of salinity for saline soils; content of organic matter for biogenic soils.

The report created for design should necessarily illustrate the methods of laboratory and field determination of soil characteristics.

The report should also be accompanied by tables and sheets of indicators of physical and mechanical characteristics of soils, diagrams of installations used in field testing, as well as columns of ground workings and engineering geological sections. Characteristics of soils should be represented by their normative values, and specific adhesion, angle of internal friction, density and ultimate strength on uniaxial compression of rocky soils, and also by design values.

The report should also include a forecast of the changes in the engineering conditions of the construction site during the construction and operation of buildings and structures. It is necessary for the design of buildings and structures to provide the original data about climatic factors and short-term meteorological loads: snow; wind; ice; fogs; other precipitation. Including data on seismic impacts.

Materials necessary for designing should be supplemented with the following information: average temperature by months; the average temperature of the coldest days; wind pressure $\mathrm{kg} / \mathrm{m}^{2}$, or $\mathrm{kPa}$ in the construction area; wind speed in $\mathrm{m} / \mathrm{s}$ according to clause 6.4 of Russian Building Codes and Regulations "SNiP" No 2.01.07-85; prevailing wind direction; snow load per $1 \mathrm{~m} 2$ at ground level $\mathrm{kg} / \mathrm{m} 2$, or $\mathrm{kPa}$; or the height of the snow cover; seismicity in points.

\section{Conclusion}

Among other variety of solar energy converters in terms of environmental friendliness and efficiency, systems that artificially reproduce the characteristics of intense atmospheric vortices, such as tornadoes and hurricanes, are the most promising. Their distribution and practical use present an undeniable prospect as one of the innovative elements of renewable energy in the 21 st century. Design and construction of the building bases and structures of solar-vortex power systems should be carried out taking into account the illustrated plan for the development of relevant technical requirements.

This research work was carried out within the framework of the State task (No. 0149-2018-0001; AAAA A16-116032810088-8; AAAA A16-116051810068-1).

\section{References}

1. G.S. Golitsyn, Study of convection with geophysical applications and analogies (Gidrometeoizdat, Leningrad, 1980)

2. A. B. Kasaeian, S. Molana, K. Rahmani, and D. Wen, Renew. Sustain. Energy Rev., 67, (2017)

3. Yu.N. Makovsky and MM Koltun, Solar Power Engineering (MIR, Moscow, 1979)

4. A. Koonsrisuk, S. Lorente, and A. Bejan, Int. J. Heat Mass Transf., 53, 1-3, (2010)

5. J. Schlaich, R. Bergermann, W. Schiel, and G. Weinrebe, ASME 2003 International Solar Energy Conference, (2003) 
6. A.I. Chabanov, V.M. Sobolev, A.A. Soloviev, M.I. Gorodov, N.M. Erokhov, and E.S. Filipenko, Inventions and useful models, 32, (2005)

7. A. Pustovgar, A.Tanasoglo, I. Garanzha, L. Shilova, A. Adamtsevich, MATEC Web of Conferences, 86, 04003 (2016)

8. A.I. Chabanov, V.M. Sobolev, and A.A. Solovyev, Patent of the Russian Federation 2265161, (2005)

9. P. Guo, Y. Wang, J. Li, and Y. Wang, Appl. Therm. Eng., 100, (2016)

10. A.A. Solovyev, Dynamic analogies in non-traditional energy (Moscow, Korina-offset Ltd., 1999)

11. A. Solovyev, A. Pustovgar, L. Shilova, A. Adamtsevich, D. Solovev, Procedia Engineering, 165, 1275 (2016) 